\title{
The Micromegas Construction Project For the ATLAS New Small Wheel
}

\author{
A. Kourkoumeli-Charalampidi* on behalf of the ATLAS Muon Collaboration \\ INFN Pavia, Italy \\ E-mail: athina.kourkoumeliepv.infn.it
}

\begin{abstract}
Large scale Micromegas (Micro mesh gaseous structure) detectors will be installed as part of the upgrade of the ATLAS innermost layer of the muon spectrometer end-cap regions, known as the New Small Wheel, in order to cope with the high radiation environment expected from the Run III onwards while providing tracking capabilities with good spatial resolution. Such a large Micromegas detector size (up to $3 \mathrm{~m}^{2}$ ) will be employed for the first time in high energy physics experiments. Each wheel will have eight detector planes, divided into two quadruplets, in order to provide tracking redundancy. Each Micromegas quadruplet will have two readout planes for the reconstruction of the precision coordinate and two with stereo reading $\left( \pm 1.5^{\circ}\right)$ for the reconstruction of the second coordinate. These detectors will provide efficiency better than $95 \%$ per single plane at rate capability up to $15 \mathrm{kHz} / \mathrm{cm}^{2}$, in presence of magnetic fields up to 0.3 T. Four different types of Micromegas quadruplets modules (SM1, SM2, LM1, LM2) each with different shape and dimensions, built by different institutes, will compose the NSW.
\end{abstract}

The 39th International Conference on High Energy Physics (ICHEP2018)

4-11 July, 2018

Seoul, Korea

${ }^{*}$ Speaker. 


\section{Introduction}

Part of the Phase-I upgrade [2] of the ATLAS experiment [1] is the replacement of the existing Small Wheels (NSW) in the end-cap region of the Muon Spectrometer with the NSW in order to maintain high performance muon tracking as well as acceptable Level-1 trigger muon fake rates in a high luminosity environment $\left(\mathscr{L}=2 \times 10^{34} \mathrm{~cm}^{-2} \mathrm{~s}^{-1}\right.$ in Run III and later on $\mathscr{L}=(5-7) \times$ $10^{34} \mathrm{~cm}^{-2} \mathrm{~s}^{-1}$ in Run IV). The NSW will feature two chamber technologies, one focusing on the Level-1 trigger function (sTGC) and one dedicated to precision tracking (Micromegas detectors) [2]-[4].

Four dimensional types of Micromegas quadruplets will be integrated in the New Small Wheel, divided into 128 quadruplets covering an active area of $1280 \mathrm{~m}^{2}$ : SM1, SM2, LM1 and LM2 to be built in Italy, Germany, France and Greece/Russia, respectively. Each wedge will be composed of two Micromegas quadruplets (see figure 1a). In order to obtain the required muon momentum resolution, the Micromegas quadruplets need to reach a spatial resolution of about $100 \mu \mathrm{m}$, which translates into stringent requirements on the mechanical accuracy of the chambers.

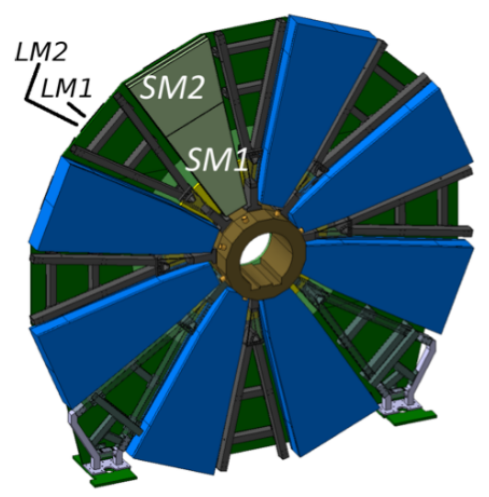

(a) NSW Micromegas quadruplets

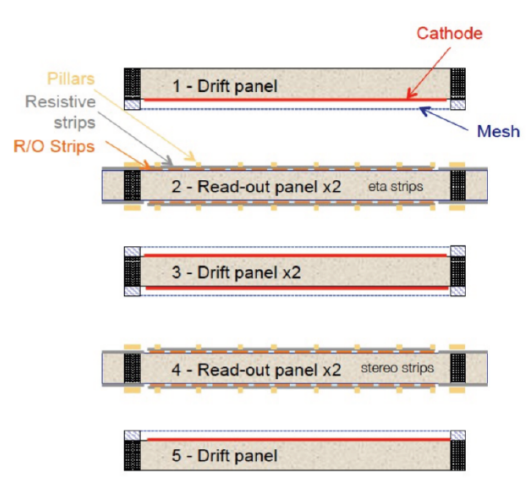

(b) The Micromegas quadruplet structure

Figure 1: The Micromegas detector quadruplets that will be part of the NSW. The SM1 and SM2 make the small wedge, while the LM1 and LM2 quadruplets make the large one. The internal structure of each quadruplet is made of drift and readout panels.

Each quadruplet is a sandwich of drift panels, containing the bare drift plane with the stretched mesh screwed and glued on top of it, and readout panels. Five panels give rise to four gas gaps, each composed by the cathode plane (bare drift panels), the readout plane and the separating micromesh. In one quadruplet there are two types of readout panels, the eta panel for reconstructing the precision coordinate (readout strips almost orthogonal to the bending tracks) and the stereo panel with $\pm 1.5^{\circ}$ strip inclination. Two types of drift panels are used; two single-layer external and a double-layer internal one positioned between the readout strips in such a way so as to create four detection layers per quadruplet (figure $1 b$ ).

Each Micromegas gas gap has two active regions, the drift region (5 $\mathrm{mm}$ high) and the amplification region $(128 \mu \mathrm{m})$. Those two regions are separated by a thin stainless steel micromesh which is made in such a way so as to provide a high transparency (about 99\%) to the electrons passing through its gaps from the drift region into the amplification region. Insulating pillars perpendicular to the resistive strips are positioned in order to maintain a gap with constant thickness $(128 \mu \mathrm{m})$ 
between the micromesh and the anodes. The detector is filled with a $\mathrm{ArCO}_{2}$ 93:7 gas mixture, while the cathode electrode is supplied with about $-300 \mathrm{~V}$ with respect to the grounded mesh. The micromesh is set to ground potential and the resistive strips are placed at positive voltage (about $570 \mathrm{~V}$ ). Due to the very high electric field in the amplification region the drifting electrons produce avalanches, with a gain of the order of $10^{4}$. In the case of the NSW Micromegas detectors the so-called floating mesh approach is utilized, which has the advantage that the amplification regions are still accessible even after the mounting of the meshes, an important feature for these large area detectors.

\section{Readout and drift panel construction}

Each readout panel is composed of six or ten Printed Circuit Board (PCB) sheets made a layer of fiber glass (FR4), resistive and $\mathrm{Cu}$ readout strips separated by a layer of insulator. A large number of pillars form a fine grating PCB sheet surface, to maintain the correct distance between the micromesh and the strips. The PCB sheets are glued on both sides of the panel, three or five PCBs per side, depending on the module size. The panel is made of aluminium frames, aluminum honeycomb sheets and cooling bars.

The construction procedure of the readout panels slightly differs in each construction site, but the principle is that the boards are built in a clean room using a granite table with a planarity guaranteed within $10 \mu \mathrm{m}$ which allows vacuum pressure to be applied on the panels and a moving stiff-back structure to close the panel. The reference surface on the table and on the stiff-back are precisely spaced by using a tapered and a V-shaped interlock, both of which allow the correct positioning of the PCBs in a XY plane.

The PCBs are first positioned facing downwards towards the aluminium plates or granite table. Half of the PCBs are placed on the granite table and the other half on a moving stiff-back structure. All ten PCBs are locked into position through pins, then placed under vacuum and at that point their planarity is measured. At this point, the gluing of the two RO planes, frames, honeycomb and cooling bars is performed. The glue is automatically disposed by a remote controlled machine both on the PCBs on the table and those on the stiff-back. The panel is closed, and the glue is left to cure. Figure 2a shows an image of a finished panel, placed on the aluminium plates of the granite table.

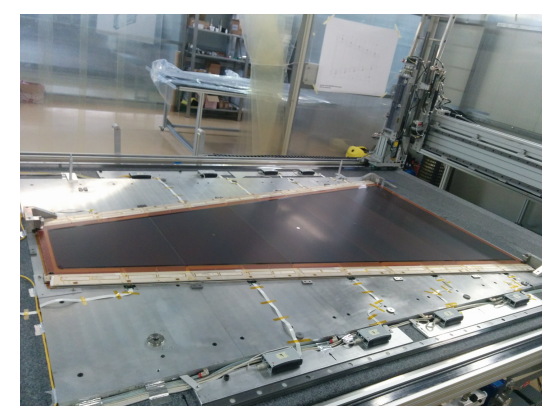

(a) Readout panel

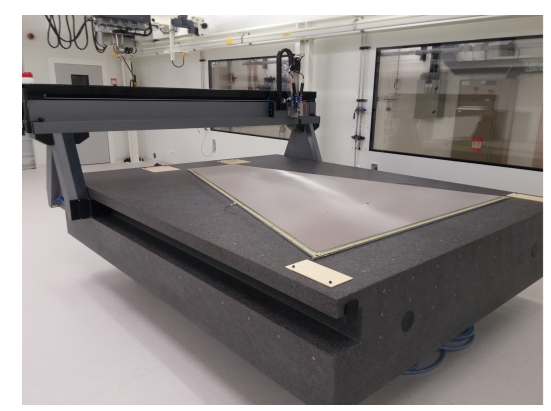

(b) Drift panel

Figure 2: A fully assembled: (a) readout panel (b) drift panel (including the mesh) 
The bare drift panels are built with the same planarity and thickness requirements of the readout panel and the procedure is very similar to that of the readout panels. On top of those panels the micromesh is glued onto $5.06 \mathrm{~mm}$ thick extruded bars which are in turn glued and screwed on the FR4 surface of the cathode sides of the bare drift panels. These bars provide the correct distance between the micromesh and the cathode surface. Before glueing the mesh all drift panels must be tested for gas tightness and all meshes and panels must be dust and dirt free. The latter is guaranteed by the panel washing, while for the former the panel surfaces are thoroughly cleaned with an electrostatic roller prior to the gap closure.

The meshes are stretched with a homogeneous tension of $\approx 10 \mathrm{~N} \mathrm{~cm}^{-1}$ using pneumatic clamps and glued to transfer frames. The additional gluing provides gas tightness between the active detector volume and the honeycomb volume. The mesh is then glued to the mesh frame while the transfer frame is removed. For the gas tightness an O-ring is used in the groove between the mesh frame and the gas gap frame. The latter defines the distance between anode and cathode and thus the drift region. Figure $2 b$ shows an image of a finished drift panel, placed on the granite table.

\section{Quadruplet assembly}

Once the five panels are constructed, they undergo a careful cleaning procedure in order to remove dust, manufacturing residues and potential greasy spots. During this phase the panels undergo a thorough cleaning and drying procedure.

Just like the panel construction, the quadruplet assembly procedure slightly differs between construction sites, but in all cases the panels are assembled in a vertical position in a clean room. One gap at a time is closed and the correct position of each panel is guaranteed by the reference pins of the stereo readout panel.

After the quadruplet is assembled (figure ??), high voltage tests are performed in order to spot any imperfections on the inside of the detector which could lead to high currents or sparks. The tests are considered successful when each sector draws less than $10 \mathrm{nA}$ in nominal voltage and there are no sparks. The high voltage tests are first performed in air (here a higher voltage is applied, which depends on the humidity levels) and then in $\mathrm{ArCO}_{2}$ 93-7 flux of $10 \mathrm{l} / \mathrm{h}$. Once those high voltage and gas tests are deemed successful, the quadruplet is transferred to the cosmic ray stand to undergo cosmic tests at the construction site, where the long term high voltage behavior of the detector is monitored.

\section{References}

[1] ATLAS Collaboration, The ATLAS Experiment at the CERN Large Hadron Collider, JINST (2008) 3 S08003

[2] ATLAS Collaboration, Technical Design Report: New Small Wheel, CERN-LHCC (2013) 006

[3] I. Giomataris et al., MICROMEGAS: A high-granularity position-sensitive gaseous detector for high particle-flux environments, Nucl. Instr. and Meth. A 376 (1996) 29

[4] ATLAS Muon collaboration, Technical Design Report: ATLAS Muon Spectrometer, CERN-LHCC (1997) 97-22 